Conclusions Herein, consistent with previous small case series, we report a good therapeutic response to RTX in patients with difficult to treat manifestations of APS. The RTX treatment protocol may affect response to therapy and a decrease of aPL titers within 2-6 months following therapy may predict a better response. Larger studies are required to confirm these results.

\section{NEUTROPHILS AND INNATE IMMUNITY IN THE PATHOGENESIS OF SLE}

1,2George Bertsias. 'Dept. of Rheumatology-Clinical Immunology, University of Crete Medical School, Heraklion; ${ }^{2}$ Institute of Molecular Biology-Biotechnology, Foundation for Research and Technology - Hellas (FORTH), Heraklion, Greece

10.1136/lupus-2020-eurolupus. 13

Background Innate immunity cells, cytokines and inflammatory pathways have been recognised as inducers and amplifiers of autoimmune responses and tissue injury in SLE.

Methods Published experimental data during the last five years on the contribution of innate immunity, particularly neutrophils and type I interferon, are summarized and their clinical implications are discussed.

Results Recent genome-wide expression studies have implicated neutrophils in human SLE and lupus nephritis. Through a combined transcriptomic, epigenetic, and functional analysis, distinct subtypes of these cells have been identified in patients with SLE, with low-density granulocytes exhibiting excessive death by generation of extracellular chromatin traps (NETs) that are decorated with immunostimulatory/alarmin molecules such as interleukin-33 and promote the activation of other immune cells, type I interferon (IFN) production and endothelial injury. Intracellular protein citrullination mediated by neutrophil peptidylarginine deiminases (PADs) is critical for NETs formation and accordingly, targeting PADs ameliorates lupus disease by reducing autoantibodies, type I IFN, immune cell activation, vascular dysfunction, and NET immunogenicity. Notably, activation of Toll-like receptors (TLR)-7/8 in neutrophils causes proteolytic cleavage of the N-terminal part of Fc$\gamma$-receptors (FcgrIIA), thus abrogating their capacity to phagocytose immunocomplexes while promoting their death by NETosis.

Mechanistically, recruitment of syntenin-1 by UNC93B1 has been reported to facilitate the sorting of TLR-7 into multivesicular bodies, therefore offering dynamic regulation of TLR-7 activation/signaling. Besides NETs, a number of other pathways may enhance IFN production in SLE such as apoptosis-derived membrane vesicles through activation of cGAS-STING, and photosensitivity-induced IFN-kappa released by keratinocytes. The latter is in line with clinical observations that therapeutic blockade of IFN or downstream signaling may be particularly beneficial in cutaneous manifestations of SLE. Remarkably, IFN signature is present in multiple immune cell types in SLE such as in B-cells where it causes breach of tolerance, promoting autoreactive $\mathrm{B}$ cell development into the autoantibodyforming cell and germinal center pathways. In T-cells, SLE patients who carry the STAT4 risk allele rs7574865[t] display augmented inflammatory responses to IL-12 and IFN- $\alpha$, and vice versa, IFN- $\alpha$ may augment the IL-12-induced STAT4 activation, therefore highlighting a subgroup of patients who may benefit from IFNa/JAK/STATs targeting.
Conclusions Culminating research further supports the critical role of neutrophils and innate immune pathways in SLE pathogenesis. Elucidation of intracellular pathways pertaining to production and regulation of important mediators such as IFN may provide novel insights towards development of targeted therapies.

Acknowledgements This work was supported by Special Research Account of the University of Crete (grant no. 4718).

\section{I14 REPEAT RENAL BIOPSY IN THE MANAGEMENT OF LUPUS NEPHRITIS}

David Jayne. Dept. of Medicine, University of Cambridge, UK

\subsection{6/lupus-2020-eurolupus. 14}

Background The therapy of lupus nephritis remains toxic and only partially effective but the benefits in terms of renal and patient survival are clear. However, there is considerable heterogeneity in the prognosis of individual patients and in their response to and tolerance of therapy. Accurate assessment of disease activity to guide therapy is limited by the complex relations between clinical biomarkers - serum creatinine, urine protein and sediment - with histologic activity. Renal biopsy is invasive with well characterised, if rare, risk of haemorrhage. While the initial biopsy confirms the diagnosis, nephritis class and other parameters, prediction of treatment response and long term outcome is limited. The extent to which the cost and risk of repeat biopsy can be justified in terms of improving long term prognosis is unclear resulting in wide variances in current practice and advice. As better targeted therapies become available in parallel with better pathway and target identification in the biopsy there is the potential role for sequential biopsy to influence the type and duration of treatment.

Methods A small number of prospective studies have examined protocol biopsies, with a larger number of observational studies limited by inconsistent indications for repeat biopsy. Outcomes have included descriptions of changes in histologic class, activity and chronicity, associations between histologic activity and clinical biomarkers, impact on treatment decisions, renal prognosis in terms of renal relapse or loss of renal function, and safety.

Results Activity on repeat protocol biopsy has been associated with increased risk of relapse and loss of function. ${ }^{1-3}$ Trends in change in histologic class have been progression from II to IV-G, from IV to IV $+V$, and for the subtypes of IV-G and IV-S to remain the same. In a small number of cases a nonlupus renal diagnosis was discovered. When compared to changes in urine protein, one third of complete proteinuric responders had histologic activity, while almost one half of non-proteinuric responder had no histologic activity. The impact of repeat biopsy on therapeutic decision remains controversial with evidence for and against.

Conclusions Repeat renal biopsy studies have contributed to our understanding of the course of nephritis and provide information unavailable from other sources. While arguments in favour of protocol biopsy continue, no long term benefit on hard outcomes has been defined. Clinical utility of repeat biopsy is highest when there is uncertainty on treatment decisions, such as, with persisting proteinuria or falling GFR, in the absence of an apparent treatment response or when 
treatment withdrawal is contemplated. Until improved blood or urine biomarkers have been developed, repeat biopsy will remain a useful tool for the clinic.

\section{REFERENCES}

1. Malvar A, Alberton V, Lococo B, et al. Kidney biopsy-based management of maintenance immunosuppression is safe and may ameliorate flare rate in lupus nephritis. Kidney Int. 2019

2. De Rosa M, Azzato F, Toblli JE, et al. A prospective observational cohort study highlights kidney biopsy findings of lupus nephritis patients in remission who flare following withdrawal of maintenance therapy. Kidney Int. 2018;94:788-794.

3. Arriens $C$, Chen $S$, Karp DR, et al. Prognostic significance of repeat biopsy in lupus nephritis: Histopathologic worsening and a short time between biopsies is associated with significantly increased risk for end stage renal disease and death. Clin Immunol. 2017;185:3-9.

\section{Oral presentations}

\section{HYDROXYCHLOROQUINE BLOOD LEVELS AND RISK OF THROMBOTIC EVENTS IN SYSTEMIC LUPUS ERYTHEMATOUS}

Maximillian F Konig, Jessica Li, Michelle Petri. Medicine, Rheumatology, Johns Hopkins University School of Medicine, Baltimore, USA

\subsection{6/lupus-2020-eurolupus. 15}

Background Hydroxychloroquine (HCQ) has a primary role in the treatment of systemic lupus erythematous (SLE). Beyond its pleiotropic immunomodulatory effects on TLR and type I interferon signaling, HCQ use has been found to be protective for thrombosis in SLE. Optimal dosing of HCQ in SLE is unknown. The longitudinal measurement of HCQ blood levels may provide an opportunity to individualize weight-based dosing strategies and reduce risk of toxicity. We examined the association of HCQ blood levels with thrombotic events in a longitudinal SLE cohort.

Methods 812 SLE patients with HCQ level measured prior to the thrombosis were included: 93\% female, 43\% AfricanAmerican, 46\% Caucasian. HCQ blood levels were quantified by liquid chromatography-tandem mass spectrometry. Mean HCQ blood levels ( \pm SD) over all cohort visits prior to occurrence of thrombosis were calculated for each patient. Thromboses were defined as venous (DVT/PE or other venous) or arterial thrombosis (stroke, myocardial infarction, digital gangrene or other arterial).

Results Thrombosis had occurred during prospective follow up in 44 patients $(5.4 \%$ ), venous in $3.0 \%$ and arterial in $2.5 \%$. Lupus anticoagulant was strongly associated with a history of any thrombosis (OR 3.25, $\mathrm{P}<0.0001$ ), venous thrombosis (OR 3.53, $\mathrm{P}<0.0001$ ), and arterial thrombosis (OR 3.08, $\mathrm{P}<0.0001)$. A prospective analysis shows that for any thrombosis and for venous thrombosis, the HCQ blood level was significantly lower. Higher prescribed doses of HCQ (as opposed to HCQ blood levels) were also associated with decreased odds of any thrombosis and venous thrombosis in a separate cross-sectional analysis (OR $0.88, \mathrm{P}=0.04$ and $\mathrm{OR}$ $0.83, \mathrm{P}=0.009$, respectively for each $1 \mathrm{mg} / \mathrm{kg}$ increase in prescribed HCQ).

Conclusions HCQ blood levels are inversely associated with risk of any thrombosis and of venous thrombosis in patients with SLE in a prospective analysis. Reduction of HCQ dosing, as suggested by the American Academy of Ophthalmologists, could reduce or eliminate the benefit of hydroxychloroquine to prevent thrombosis.
Acknowledgements The Hopkins Lupus Cohort is supported by NIH Grant RO1 AR 69572.

\section{O2 EFFECT OF TREATMENT ON ANTIPHOSPHOLIPID ANTIBODIES IN SLE}

${ }^{1}$ Michelle Petri, ' Laurence S Magder, 'Daniel W Goldman. 'Medicine, Rheumatology, Johns Hopkins University School of Medicine, Baltimore; ${ }^{2}$ Dept. Epidemiology, University of Maryland, School of Medicine, Baltimore, USA

\subsection{6/lupus-2020-eurolupus. 16}

Background Unlike primary antiphospholipid syndrome patients, most SLE patients with antiphospholipid antibodies are on one or more treatments for their SLE that might affect levels of their antiphospholipid antibodies. We examined the effect of prednisone and hydroxychloroquine on antiphospholipid antibodies in an SLE longitudinal cohort.

Methods 943 SLE patients, who were tested for each anticardiolipin isotype (aCL $\operatorname{IgG}, \operatorname{IgM}$ and $\operatorname{IgA}$; INOVA) and lupus anticoagulant (LAC; dRVVT with further confirmatory testing) for at least 10 quarterly clinic visits, were included. We compared visits positive for antiphospholipid antibodies (aCL $>20$ and aCL $>40$; dRVVT $>45$ ) to visits negative for antiphospholipid antibodies, with respect to treatment, using conditional logistic regression and conditioning on the patient.

Results Prednisone treatment significantly reduced the levels of aCL IgG isotypes (aCL IgG $>40$ and some prednisone but less than $10 \mathrm{mg} /$ day, OR $0.6195 \%$ CI $0.46-0.80 \mathrm{p}=0.0004$ ), but not aCL IgM, IgA, or LAC. Hydroxychloroquine treatment significantly reduced aCL IgG (aCL IgG $>40$, OR $0.3595 \%$ CI $0.22-0.55 \mathrm{p}<0.0001)$, aCL IgM (aCL IgM>40, OR 0.56 95\% CI $0.36-0.87 \mathrm{p}=0.010$ ) and LAC (OR $0.71 \quad 95 \%$ CI $0.58-0.86 \mathrm{p}=0.007)$ but not aCL IgA.

Conclusions Prednisone does not reduce IgM or IgA isotypes of anticardiolipin, or LAC. These results explain why prednisone does not reduce thrombosis in SLE. Hydroxychloroquine, on the other hand, significantly reduces all antiphospholipid types except for the IgA isotype of anticardiolipin. This may explain why IgA isotypes are more common in SLE. It may also explain why hydroxychloroquine leads to only a $50 \%$ reduction in thrombosis, as IgA isotypes do confer some risk of thrombosis.

Acknowledgements The Hopkins Lupus Cohort is supported by NIH Grant RO1 AR 69572.

\section{O3 CHANGES IN GUT MICROBIOTA AFTER SYNBIOTIC SUPPLEMENTATION IN PATIENTS WITH SYSTEMIC LUPUS ERYTHEMATOSUS: A RANDOMISED, DOUBLE- BLIND, PLACEBO-CONTROLLED TRIAL}

\footnotetext{
${ }^{1,2,3}$ Alvina Widhani, ${ }^{1,2}$ Samsuridjal Djauzi, ${ }^{4}$ Fransiscus D Suyatna, ${ }^{5}$ Beti Ernawati Dewi, ${ }^{4}$ Melva Louisa, ${ }^{5}$ Andi Yasmon, ${ }^{6}$ Susan Rahayu. ${ }^{1}$ Allergy and Clinical Immunology Division, Dept. of Internal Medicine, Faculty of Medicine, Universitas Indonesia, Jakarta; ${ }^{2}$ Dr. Cipto Mangunkusumo National General Hospital, Jakarta; ${ }^{3}$ Doctoral Program in Biomedical Science, Faculty of Medicine, Universitas Indonesia, Jakarta; ${ }^{4}$ Dept. of Pharmacology and Therapeutic, Faculty of Medicine, Universitas Indonesia, Jakarta; ${ }^{5}$ Dept. of Microbiology, Faculty of Medicine, Universitas Indonesia, Jakarta; ${ }^{6}$ Molecular Biology Unit, Integrated Laboratory, Faculty of Medicine, Universitas Indonesia, Jakarta, Indonesia
}

10.1136/lupus-2020-eurolupus. 17 